\title{
Assessing quality with qualitative research
}

\author{
Jill M. Mhyre, MD
}

Published online: 6 March 2010

(c) Canadian Anesthesiologists' Society 2010

Epidural analgesia is currently the most effective method available to control pain in labour and has become a popular choice for labouring women in North America. In Canada, epidural analgesia was used in nearly half (45.7\%) of all vaginal deliveries in 2001-2002. ${ }^{1}$ In the United States, a sample from seven states suggests that $68 \%$ of women received neuraxial labour analgesia in $2003 .^{2}$

Ideally, labour analgesia should be tailored to the individual patient's labour, medical condition, preferences, and goals. ${ }^{3}$ To this end, current literature most clearly addresses interactions between neuraxial labour analgesia and the progress of labour, mode of delivery, complication rates, and a number of medical conditions. In comparison, less evidence is available to measure the effectiveness of various analgesic techniques in addressing women's preferences and goals for analgesia during labour and delivery.

Maternal satisfaction with pain relief is a common measure used in quantitative trials of various analgesic techniques in order to capture effectiveness in meeting women's expectations, preferences, and goals. However, satisfaction is a multidimensional construct influenced by analgesic experience, delivery outcomes and complications, human interactions, and other factors. ${ }^{4}$ As a result, a global measure of satisfaction with analgesia may or may not discriminate successfully between a high-quality and a low-quality analgesic experience.

The limitation of relying on global satisfaction to signal high-quality analgesia is apparent when considering the

J. M. Mhyre, MD $(\bowtie)$

Department of Anesthesiology, University of Michigan Health

System, Room L3622 Women's Hospital, 1500 East Medical

Center Drive, Ann Arbor, MI 48109-5278, USA

e-mail: jmmhyre@umich.edu; jmmhyre@med.umich.edu group of randomized controlled trials that compared epidural analgesia with non-epidural analgesia or no analgesia. ${ }^{5}$ Five of these trials included a measure of satisfaction with analgesia among the outcomes, and metaanalysis suggests no significant difference between groups (relative risk 1.18, 95\% confidence interval 0.92 to 1.50 ; $n=1,940$ women).

In pursuit of a more robust method to evaluate the factors that determine high-quality neuraxial analgesia from women's perspectives, Dr. Pamela Angle and her colleagues have initiated an ambitious program of mixedmethods research. Published in the current issue of the Journal, their article describes a series of interviews and focus groups that aimed to identify themes relevant to the experience of childbirth pain and epidural analgesia. ${ }^{6}$ The purpose of this qualitative study was to define the specific factors that may be important for an overall measure of high-quality analgesia. Participants were women of mixed parity who had received epidural labour analgesia and who had met with the investigators to discuss their experience of epidural labour analgesia within $72 \mathrm{hr}$ following delivery.

The major themes that emerged suggest women are concerned primarily that effective analgesia should be readily available, free of effects on labour progress, and established without complications. If trade-offs become necessary, rather than avoidance of other side effects, such as pruritis, continuous pain control is most important, followed by preserved motor strength and mobility. Beyond this, a high-quality analgesic experience should: 1 ) restore a feeling of internal control and the ability to focus; 2 ) enable decision-making and give back (external) control; 3) preserve desirable bodily sensations of labour progress; 4) preserve mobility and strength; and 5) improve a woman's overall labour and delivery experience. 
These findings seem reasonable and have face validity. However, readers familiar with quantitative science may struggle to determine whether this qualitative study itself was of high quality. Methods used to appraise quantitative research do not translate well to qualitative studies, potentially leading some readers to consider the reported study as being either "soft" or of uncertain substance. The remainder of this editorial outlines a strategy for reading qualitative studies and considers the strengths and limitations of the current study of women's perspectives on quality labour epidural analgesia.

Qualitative research includes an extensive portfolio of approaches representing a broad cohort of scientific traditions. As a result of this heterogeneity, quality checklists of specific research procedures (modeled after the CONSORT criteria) are impossible to construct for qualitative research. ${ }^{7}$ Instead, the evaluation of qualitative research relies on careful consideration of the appropriateness and usefulness of the study. A clearly articulated research purpose should drive the study design, execution, analysis, and presentation..$^{7-11}$ In addition, qualitative research should be ethical, important, clearly and coherently articulated, and use rigorous methods for data collection and analysis, ${ }^{10}$ all standards that the article published in this issue appears to have met. Finally, validity is an essential characteristic of high-quality qualitative research. However, a specific understanding of the meaning of validity depends on the researcher's philosophy of science, and philosophies vary across research traditions.

The most common philosophical perspectives that underlie contemporary science include positivistism and constructivism, also known as interpretivism. These perspectives are distinguished by their ontologic and epistemologic positions. Where ontology is the philosophical study of the nature of being, existence, or reality in general, epistemology refers to the philosophical study of the nature of knowledge, what constitutes knowledge, and how knowledge is acquired. ${ }^{11}$

The positivist position is most familiar to anesthesiologists because it underlies the quantitative scientific tradition of medicine. Positivist ontology assumes that a unified reality exists, and the corresponding epistemology posits that reality can be discovered through robust application of the scientific method. While an accurate description of reality is an initial goal for the positivist, discovery is achieved most rigorously by constructing hypotheses and testing them using pre-defined logical operations (most commonly statistical inference), which are applied to empirical data collected through the objective observation of phenomena.

Qualitative researchers working in the positivist tradition aim to engage deeply in phenomena in order to record precisely evidence that may be used to describe or explain a unified external reality. Validity or credibility depends on accurate recording and interpretation, each of which may be enhanced through procedures such as triangulation, disinterested peer-review, external audits, and member checking. Triangulation is the qualitative research technique in which the same question is approached using a variety of techniques and sources of data. The study by Angle et al. triangulated the results from both the focus groups and the individual interviews to generate data. Rigor was further strengthened by use of a memberchecking procedure in which the investigator's interpretation of findings was verified with participants at the end of each focus group and interview. In addition, the interviews and focus groups were audiotaped and transcribed verba$\mathrm{tim}$, and two investigators (an anesthesiologist and a labour nurse researcher) coded the transcripts independently before comparing codes and emerging themes. Professional bias was managed by forming an interdisciplinary research team that included not only an anesthesiologist but also several nurses and a medical social scientist with expertise in qualitative research methodology. The themes that emerged were derived inductively from the data.

Constructivists argue that a universal external reality either does not exist or cannot be known, that contextually embedded reality is constructed intersubjectively, and that concepts, models, and theories are viable (as opposed to "true") to the extent that they prove useful for the contexts in which they were created. ${ }^{12}$ Based on this view, the purpose of science is to engage deeply in phenomena, to capture and preserve empirical material relevant to the phenomena of interest, and to construct understanding based on individual interpretations of the empirical material and dialogue between engaged parties. ${ }^{10}$ Rather than attempt to expunge bias, constructivists either harness investigator bias (the investigator is seen as an instrument of data collection) or manage bias through active reflection. ${ }^{7,10}$ A priori hypotheses are constructed, not to be tested quantitatively, but to define pre-existing bias clearly and to serve as a starting point for the reflective process.

The constructivist position recognizes that the discussion of epidural analgesic quality is fundamentally a social act. Since women could freely decline to meet with the investigator (a design choice that enhanced the ethical rigor of the study), only those willing to engage in this social process made a contribution to the findings. The interviews and focus groups were designed, conducted, and analyzed by an obstetric anesthesiologist. Even in the absence of a direct clinical relationship, participants were almost certainly aware of the investigator's clinical role. Interviews were conducted within $72 \mathrm{hr}$ following delivery, a time that may be associated with a "halo effect" in which a woman's assessments may be influenced by the overriding joy of just having given birth successfully. ${ }^{13}$ The study balanced these 
social forces with a series of questions in the interview guide that encouraged criticism, i.e., asking about the aspects that caused worry, bother, or areas for improvement, and requesting a description of the ideal epidural for labour and delivery.

From the constructivist perspective, the results reported by Dr. Angle et al. represent a distillation of the elements of high- and low-quality analgesia expressed within this particular social context. Rather than being seen as a source of bias, the expertise of an obstetric anesthesiologist was critical for eliciting detailed reflections on the analgesic experience and for recognizing how these reflections may be useful to inform future analgesic techniques. Patient reflections offered only a partial answer; it was the discourse between interested parties that generated a useful and valid result.

As with quantitative research, qualitative studies can be more or less externally valid or generalizable. Given the widespread popularity of epidural analgesia, these results may be considered to be widely applicable. Nevertheless, specific characteristics of the participants were important. All but one participant had planned or considered epidural analgesia before labour. The participants were not only well educated but also relatively wealthy. All but one participant had a partner, and all delivered in birthing units in the greater Toronto area. None of the participants had experienced a perinatal loss, major perinatal morbidity, or major anesthetic or obstetric maternal complication.

To both evaluate and expand external generalizability, this investigation could be repeated in additional patient populations by investigators with different professional backgrounds and could even be repeated between the original investigator and her participants. Women's assessments have been shown to evolve as they integrate their experiences over months to years. ${ }^{13}$ Women who enter labour with the intention of an unmedicated birth may have a different perspective on the role and benefits of epidural analgesia. Once the qualitative study results are translated into a survey instrument, then mixedmethods research, which combines both qualitative and quantitative methods, may be used to further triangulate results.

Relief from pain is only one of numerous factors that contribute to the analgesic experience. Additional factors articulated in this study include availability, safety, and the patient's perception of internal and external control while minimizing undesirable side effects. A psychometric instrument that measures some or all of these components may become a useful tool to measure the outcome of analgesic quality for future trials comparing neuraxial analgesic techniques.

\section{L'évaluation de la qualité à l'aide de la recherche qualitative}

L'analgésie péridurale est, à l'heure actuelle, la méthode la plus efficace à notre disposition pour soulager la douleur pendant le travail obstétrical. Elle est devenue un choix populaire pour les femmes en travail en Amérique du Nord. Au Canada, l'analgésie péridurale a été pratiquée dans presque la moitié $(45,7 \%)$ des accouchements par voie vaginale réalisés en 2001-2002. ${ }^{1}$ Aux États-Unis, un échantillon portant sur sept états suggère que $68 \%$ des femmes ont bénéficié d'une analgésie neuraxiale pour le travail obstétrical en $2003 .^{2}$

Dans l'idéal, l'analgésie pour le travail obstétrical devrait être adaptée au travail particulier de chaque patiente, selon son état pathologique, ses préférences et ses objectifs. $^{3}$ À cet effet, la littérature actuelle s'intéresse principalement à des questions liées aux interactions entre l'analgésie neuraxiale pour le travail obstétrical et la progression du travail, le type d'accouchement, les taux de complications et différents états pathologiques. Par contre, nous ne disposons que de peu de données probantes mesurant l'efficacité de diverses techniques analgésiques pour répondre aux préférences et aux objectifs des femmes en matière d'analgésie pendant le travail obstétrical et l'accouchement.

La satisfaction de la mère en matière de soulagement de la douleur est une mesure fréquemment utilisée dans les études quantitatives comparant plusieurs techniques d'analgésie. Cette mesure permet de saisir l'efficacité d'une technique donnée à répondre aux attentes, aux préférences et aux objectifs des femmes. Toutefois, la satisfaction est un construit pluridimensionnel influencé par l'expérience de la patiente avec les analgésiques, les résultats de l'accouchement et les complications, les interactions humaines et bien d'autres facteurs. ${ }^{4}$ Dès lors, une mesure globale de la satisfaction en matière d'analgésie pourrait ou non permettre d'établir une distinction claire entre une expérience analgésique de bonne ou de mauvaise qualité.

Les limites d'une mesure de la satisfaction globale pour évaluer une analgésie de bonne qualité sont évidentes lorsqu'on examine le groupe d'études randomisées contrôlées qui ont comparé l'analgésie péridurale à l'analgésie non péridurale et à pas d'analgésie. ${ }^{5}$ Dans cinq de ces études, les critères d'évaluation comprenaient une mesure de la satisfaction par rapport à l'analgésie, et une méta-analyse a suggéré qu'il n'y avait pas de différence significative entre les groupes (risque relatif 1,18 , intervalle de confiance $95 \% 0,92$ à $1,50, n=1940$ femmes). 
Dans leur recherche d'une méthode plus fiable d'évaluation des facteurs qui définissent une analgésie neuraxiale de bonne qualité selon les femmes, Dr Angle et ses collègues ont lancé un programme ambitieux de recherche avec des méthodes mixtes. Dans ce numéro du Journal, leur article décrit une série d'entretiens et de groupes de discussion qui avaient pour objectif d'identifier les thèmes pertinents à l'expérience de la douleur de l'accouchement et à l'analgésie péridurale. ${ }^{6}$ Le but de cette étude qualitative était de déterminer quels facteurs spécifiques jouaient possiblement un rôle de premier plan dans la mesure globale d'une analgésie de bonne qualité. Des femmes de parités diverses ayant reçu une analgésie péridurale pour le travail ont participé à l'étude. Elles ont rencontré les chercheurs afin de discuter de leur expérience par rapport à l'analgésie péridurale dans les 72 heures suivant l'accouchement.

Les thèmes principaux qui sont apparus suggèrent que les préoccupations premières des femmes sont les suivantes : une analgésie efficace doit être immédiatement disponible, ne pas avoir d'effets sur la progression du travail, et se faire sans complications. Dans les cas où il faut faire des compromis, le contrôle continu de la douleur devient alors l'élément primordial, suivi par la conservation de la force motrice et de la mobilité, plus que le fait d'éviter d'autres effets secondaires comme le prurit. Au-delà de ces exigences, une expérience analgésique de bonne qualité devrait: 1) redonner à la patiente un sentiment de contrôle interne et la capacité de se concentrer; 2) permettre la prise de décision et redonner un contrôle [externe]; 3) préserver les sensations corporelles désirables concernant la progression du travail; 4) conserver la mobilité et la force; et 5) améliorer l'expérience globale du travail et de l'accouchement.

De tels résultats semblent raisonnables et ont une validité apparente. Toutefois, les lecteurs aguerris à la science quantitative pourraient avoir de la difficulté à décider si cette étude qualitative était en soi de bonne qualité. Les méthodes utilisées pour juger de la qualité de recherches quantitatives sont maladroites lorsqu'elles sont appliquées à des études qualitatives, ce qui pourrait mener certains lecteurs à juger l'étude présentée ici comme de substance incertaine ou «peu solide ». Le reste de cet éditorial s'emploiera à décrire une stratégie de lecture des études qualitatives et examinera les forces et les faiblesses de l'étude d'Angle et coll. sur le point de vue des femmes concernant une analgésie péridurale de qualité pour le travail obstétrical.

La recherche qualitative dispose d'un vaste portefeuille d'approches, lesquelles sont le reflet de la diversité des traditions scientifiques. En raison de cette hétérogénéité, des listes de contrôle de qualité définissant des procédures de recherche spécifiques (suivant le modèle des critères
CONSORT) sont impossibles à établir en recherche qualitative. $^{7} \mathrm{Au}$ lieu de cela, l'évaluation de la recherche qualitative s'appuie sur un examen approfondi de l'utilité et de la justesse d'une étude. Un objectif de recherche clairement articulé devrait être à l'origine de la conception de l'étude, de son exécution, de son analyse et de sa présentation. ${ }^{7-11}$ De plus, une recherche qualitative se doit d'être déontologique, importante, articulée de façon claire et cohérente, et d'utiliser des méthodes rigoureuses de collecte et d'analyse des données ${ }^{10}$ - des normes que l'article publié ici semble avoir respectées. Enfin, la validité constitue un élément essentiel d'une recherche qualitative de bonne qualité. Cependant, la compréhension particulière de ce qu'on entend par «validité » dépend de la philosophie scientifique du chercheur, et ces philosophies divergent d'une tradition de recherche à une autre.

Parmi les perspectives philosophiques les plus courantes dans la science actuelle, il y a le positivisme et le constructivisme, aussi connu sous le nom de relativisme. Ces perspectives se distinguent par leurs positions ontologiques et épistémologiques. Alors que l'ontologie est l'étude philosophique de la nature de l'être, de l'existence ou de la réalité en général, l'épistémologie fait référence à l'étude philosophique de la nature de la connaissance, de ce qui constitue la connaissance, et de la manière dont celle-ci est acquise. ${ }^{11}$

La position positiviste est celle que les anesthésiologistes connaissent le mieux car il s'agit de la philosophie qui sous-tend la tradition scientifique quantitative de la médecine. L'ontologie positiviste prend pour acquis qu'une réalité unifiée existe, et l'épistémologie correspondante affirme que la réalité peut être révélée grâce à une application rigoureuse de la méthode scientifique. Alors qu'une description fidèle de la réalité est un but initial pour le positiviste, la découverte est réalisée de la façon la plus rigoureuse en élaborant des hypothèses et en les testant à l'aide d'opérations logiques prédéfinies (en général par inférence statistique), opérations qui sont appliquées à des données empiriques récoltées par l'observation objective de phénomènes.

Le but des chercheurs qualitatifs travaillant dans la tradition positiviste est de s'immerger dans les phénomènes de façon à enregistrer précisément des données probantes qui pourraient être utilisées pour décrire ou expliquer une réalité extérieure unifiée. La validité ou la crédibilité dépendent alors d'une mesure et d'une interprétation précises des données, ces deux composantes pouvant être améliorées grâce à des processus tels que la triangulation, la révision impartiale par les pairs, des vérifications externes ou encore la validation par les participants. La triangulation est une technique de recherche qualitative par laquelle la même question est abordée à l'aide de toutes sortes de techniques et de sources de données. L'étude de 
Dr Angle et coll. a triangulé les résultats des groupes de discussion et des entretiens individuels afin de générer des données. En outre, la rigueur a été encore plus poussée en raison du recours à une méthode de validation par les participantes : en effet, l'interprétation des chercheurs a été par la suite vérifiée par les participantes à la fin de chaque groupe de discussion et de chaque entretien. De plus, les entretiens et les groupes de discussion ont fait l'objet d'un enregistrement sonore et ont été retranscrits mot pour mot, et deux chercheurs (une anesthésiologiste et une infirmière de recherche en travail obstétrical) ont codé de façon indépendante les transcriptions avant de comparer les codes et de faire émerger les thèmes. Le biais professionnel a été mitigé grâce à la formation d'une équipe de recherche interdisciplinaire composée non seulement d'une anesthésiologiste mais également de plusieurs infirmières et d'un scientifique social médical ayant de l'expérience dans la méthodologie de recherche qualitative. Les thèmes qui sont apparus ont été dérivés des données par induction.

Selon l'opinion constructiviste, soit il n'existe pas de réalité extérieure universelle, soit elle ne peut être connue; une réalité intégrée dans un contexte est construite de façon intersubjective et les concepts, modèles et théories sont viables (plutôt que 'vrais') dans la mesure où ils sont utiles aux contextes dans lesquels ils ont été créés. ${ }^{12} \mathrm{Si}$ l'on se fonde sur ce point de vue, l'objectif de la science est de s'immerger dans les phénomènes, de saisir et de conserver du matériel empirique pertinent aux phénomènes à l'étude, et de construire une compréhension qui s'appuie sur les interprétations individuelles de ce matériel empirique et le dialogue entre les parties impliquées. ${ }^{10}$ Plutôt que d'essayer d'effacer un biais, les constructivistes vont soit exploiter le biais du chercheur (le chercheur est perçu comme un instrument de collecte de données) ou gérer le biais par une réflexion active. ${ }^{7,10}$ Des hypothèses a priori sont construites non pas dans le but d'être testées quantitativement, mais afin de clairement définir le biais préexistant et servir de point de départ au processus réflexif.

Le point de vue constructiviste reconnaît que le fait de discuter de la qualité d'une analgésie péridurale est, fondamentalement, un acte social. Les femmes pouvant librement refuser de rencontrer le chercheur (un choix de méthodologie qui a amélioré la rigueur éthique de l'étude), seules celles qui désiraient participer à ce processus social ont apporté leur contribution aux résultats de l'étude. Les entretiens et les groupes de discussion ont été conçus, menés et analysés par une anesthésiologiste spécialisée en obstétrique. Même en l'absence d'une relation clinique directe, les participantes étaient très probablement conscientes du rôle clinique de la chercheuse. Les entretiens ont été réalisés au cours des 72 heures suivant l'accouchement, une période qui pourrait impliquer un «effet de halo», pendant laquelle les jugements d'une femme pourraient être influencés par la joie incommensurable d'avoir donné naissance. ${ }^{13}$ L'étude a contrebalancé ces forces sociales en présentant aux participantes une série de questions destinées à encourager la critique, en posant par exemple des questions sur les aspects de l'analgésie qui ont provoqué de l'inquiétude, des préoccupations ou des choses à améliorer, et en leur demandant de décrire la péridurale idéale pour le travail obstétrical et l'accouchement.

D'un point de vue constructiviste, les résultats rapportés par Dr Angle et coll. représentent une distillation des éléments d'une analgésie de bonne et de mauvaise qualité tels qu'exprimés dans ce contexte social en particulier. Plutôt que d'être perçue comme une source de biais, l'expertise d'une anesthésiologiste spécialisée en obstétrique a joué un rôle critique pour mettre au jour des réflexions détaillées sur l'expérience de l'analgésie, et pour reconnaître l'utilité possible de ces réflexions dans l'élaboration de techniques analgésiques futures. Les réflexions des patientes n'ont fourni qu'une réponse partielle; c'est le dialogue entre les parties impliquées qui a permis de générer un résultat utile et valide.

Tout comme c'est le cas avec la recherche quantitative, les études qualitatives peuvent être plus ou moins valides ou généralisables d'un point de vue externe. Étant donné la grande popularité de l'analgésie péridurale, on peut considérer que ces résultats peuvent s'appliquer à de nombreux cas. Néanmoins, il importe de souligner certaines caractéristiques spécifiques des participantes de cette étude. Toutes sauf une avaient envisagé ou planifié de recevoir une analgésie péridurale avant le travail obstétrical. Non seulement les participantes étaient bien scolarisées, mais elles étaient également relativement aisées. Toutes sauf une avaient un partenaire, et toutes ont accouché dans des unités de naissance de la région du Grand Toronto. Aucune des participantes n'avait subi de perte périnatale, de morbidité périnatale majeure, ou de complication maternelle anesthésique ou obstétricale majeure.

Si l'on souhaitait évaluer et étendre la dimension généralisable à l'externe de cette étude, elle pourrait être répétée dans d'autres populations de patientes, par des chercheurs possédant des antécédents professionnels différents, ou même entre la chercheuse originale et ses participantes. Il a été démontré que la perspective des femmes évoluait au fil du temps, au fil de l'intégration de leurs expériences. ${ }^{13}$ Les femmes commençant le travail obstétrical avec l'intention d'accoucher sans aide médicamenteuse pourraient avoir une perspective bien différente du rôle et des avantages d'une analgésie péridurale. Une fois que les résultats d'une étude qualitative seront traduits dans un instrument de sondage, 
des recherches à méthodes mixtes combinant les méthodes qualitative et quantitative pourront être utilisées pour trianguler les résultats de façon plus approfondie.

Le soulagement de la douleur n'est qu'un facteur parmi d'autres contribuant à l'expérience analgésique. Parmi les autres facteurs que cette étude a fait ressortir, citons la disponibilité, la sécurité, et la perception de contrôle interne et externe de la patiente, outre la minimisation des effets secondaires indésirables. Un instrument psychométrique mesurant certaines ou toutes ces composantes pourrait s'avérer utile pour mesurer les résultats de la qualité de l'analgésie dans des études futures comparant les diverses techniques d'analgésie neuraxiale.

Support The author is employed by the University of Michigan Health System. No external sources of funding were provided. There are no conflicts of interest to disclose.

\section{References}

1. Errata-Giving Birth in Canada: A Regional Profile. Canadian Institute for Health Information, 2005. Available from URL: http://secure.cihi.ca/cihiweb/en/downloads/GBC2004_errata_e.pdf (accessed February 2010).

2. Martin JA, Menacker F. Expanded health data from the new birth certificate, 2004. Natl Vital Stat Rep 2007; 55: 1-22.

3. Wong C. Epidural and spinal analgesia/anesthesia for labor and vaginal delivery. In: Chestnut DH, Polley LS, Tsen LC, Wong
CA (Eds). Chestnut's Obstetric Anesthesia Principles and Practice, $4^{\text {th }}$ ed. Philadelphia: Mosby; 2009; 4: 479.

4. Robinson PN, Salmon P, Yentis SM. Maternal satisfaction. Int J Obstet Anesth 1998; 7: 32-7.

5. Anim-Somuah M, Smyth R, Howell C. Epidural versus non-epidural or no analgesia in labour. Cochrane Database Syst Rev 2005; (4): CD000331.

6. Angle P, Kurtz Landy C, Charles $C$, et al. Phase 1 Development of an index to measure quality neuraxial labor analgesia: exploring childbearing women's perspectives. Can J Anesth 2010; 57. DOI: $10.1007 / \mathrm{s} 12630-010-9289-1$.

7. Stige B, Malterud K, Midtgarden T. Toward an agenda for evaluation of qualitative research. Qual Health Res 2009; 19: 1504-16.

8. Malterud K. Qualitative research: standards, challenges, and guidelines. Lancet 2001; 358: 483-8.

9. Public Health Resource Uni. 10 questions to help you make sense of qualitative research. In: Critical Appraisal Skills Programme (CASP): Making Sense of Evidence, 2006. Available from URL: http://www.phru.nhs.uk/Doc_Links/Qualitative\%20Appraisal\% 20Tool.pdf (accessed February 2010).

10. Cohen DJ, Crabtree $B F$. Evaluative criteria for qualitative research in health care: controversies and recommendations. Ann Fam Med 2008; 6: 331-9.

11. Mason J. Qualitative Researching. 2nd ed. Newbury Park: SAGE Publications; 2002.

12. Vonglasersfeld E. A constructivist approach to teaching. In: Steffe LP, Gale J (Eds). Constructivism in Education. Mahwah, NJ: Lawrence Erlbaum Associates; 1995: 3-15.

13. Simkin P. Just another day in a woman's life? Part II: Nature and consistency of women's long-term memories of their first birth experiences. Birth 1992; 19: 64-81. 